American Journal of Applied Sciences 9 (5): 759-765, 2012

ISSN 1546-9239

(C) 2012 Science Publications

\title{
Automatic Palmprint Identification based on High Order Zernike Moment
}

\author{
${ }^{1}$ Gayathri, R. and ${ }^{2}$ P. Ramamoorthy \\ ${ }^{1}$ Department of Electronics and Communication Engineering, \\ Vel Tech Dr. RR and Dr. SR Technical University, Chennai, India \\ ${ }^{2}$ Department of Electronics and Communication Engineering, \\ Sri Shakthi Institute of Engineering Technology, Coimbatore, India
}

\begin{abstract}
Problem statement: Hand geometry contains relatively invariant features of an individual. Palmprint recognition is an efficient biometric solution for authentication system. The existence of several hand-based authentication commercial systems indicates the effectiveness of this type of biometric. Approach: We proposed a palmprint verification system using high order Zernike moment that was robust to rotation, translation and occlusion. Zernike moment was an efficient algorithm for representing the shape features of an image. The design consists of feature extraction and matching of image using high order Zernike moment. Zernike moments at high orders was calculated from the image and the image was classified using K-Nearest Neighborhood (KNN). The reason for using Zernike moment was that it was the best algorithm due to its orthogonality and rotation invariance property. Results and Conclusion: Computational cost can be reduced by detecting the common term of Zernike moment. Experiments and classifications have been performed using Hong Kong PolyU palm print database with 125 individuals' left hand palm images; every person has 5 samples, totaling up to 625 . We then get every person's palm images as a template (totaling 125). The remaining 500 are used as the training samples. The proposed palmprint authentication system achieves a recognition accuracy of $98 \%$ and interesting working point with False Acceptance Rate (FAR) of $=1.062 \%$ and False Rejection Rate (FRR) of $=0 \%$. Experimental evaluation demonstrates the efficient recognition performance of the proposed algorithm compared with conventional palmprint recognition algorithms.
\end{abstract}

Key words: Zernike moment, KNN classification, computational cost, recognition, conventional palmprint, recognition algorithms, false rejection rate, efficient algorithm, classified using, reduced by detecting, common term

\section{INTRODUCTION}

A biometric recognition system is essentially a pattern recognition system that acquires biometric data from an individual, extracts a salient feature set from the data,compares this feature set against the feature set(s) stored in the database and executes an action based on the result of the comparison (Krishneswari and Arumugam, 2012; MeenakshiDevi et al., 2009; Breu et al., 1995; Al-Hamami and Ani, 2005; Basha et al., 2011).

In mathematics, Zernike moment is a sequence of polynomials over a unit disk. It is named after Frits Zernike; it plays an important role in beam optics. Moment functions are used to capture global features of the image in pattern recognition and image analysis (Prokop and Reeves, 1992). Zernike moments are used in a wide range of applications on image analysis as they have minimum redundancy (Teague, 1980) and rotation invariance.
A palmprint image is uniquely determined by its geometrical moments of all orders. Low-order moments contain less information, while high-order moments are vulnerable to noise. The use of orthogonal moments makes it possible to describe a palmprint image with a 'finite number of moments and get benefit from the inclusion of high-order moments'. However, there are also some technical difficulties in the calculation of Zernike moments due to the very high computational complexity and lack of numerical precision. It is usually not possible to calculate them accurately in reasonable time when the desired moment order is high and/or the images to be processed are large. Little attention has been paid to the efficient and accurate calculation of Zernike moments (Mukundan and Ramakrishnan, 1995). (Mukundan and Ramakrishnan, 1995) proposed a recursive algorithm for computing the Zernike and Legendre moments in polar coordinates.

Corresponding Author: Gayathri, R ., Department of Electronics and Communication Engineering,

Anna University of Technology, Coimbatore, Tamil Nadu, India Tel: 919884811501 
Am. J. Applied Sci., 9 (5): 759-765, 2012

As a final point a more recent study, by (Gu et al., 2002) employed the square to circular transformation of Mukundan and Ramakrishnan (1995) and more efficient recursive relations to develop an even faster algorithm but its accuracy is still limited when compared with that of (Mukundan and Ramakrishnan, 1995) because of approximate coordinate transformation.

\section{MATERIALS AND METHODS}

Moment functions of image intensity values are used to capture global features of the image in pattern recognition and image analysis (Teh and Chin, 1988). Zernike moments are used in various applications in image analysis. When compared to various moment based descriptors, Zernike moments have the advantages of minimal redundancy, rotation invariance and robustness to noise.

Zernik polynomials were introduced by $\mathrm{F}$. Zernike. Zernike moments are a sequence of orthogonal polynomial inside a unit disk. Based on a set of complex polynomials that form a complete orthogonal set over the interior of the unit circle (Khotanzad, 1990).

They are defined to be the projection of the image function on these orthogonal basis functions.

The Zernike polynomial of order $q$ and repetition $p$ can be described by the following Eq. 1:

$\mathrm{V}_{\mathrm{q}, \mathrm{p}}(\mathrm{x}, \mathrm{y})=\mathrm{V}_{\mathrm{q}, \mathrm{p}}(\rho, \varphi)=\mathrm{R}_{\mathrm{q}, \mathrm{p}}(\rho) \mathrm{e}^{\mathrm{j} p \varphi^{\prime}}$

where $\mathrm{q}$ is a positive integer or zero and $\mathrm{p}$ is an integer, subject to the following constraints $q-|p|$ is even and $\mathrm{q}<|\mathrm{p}|, \rho$ is the length of the vector from origin to ( $\mathrm{x}$, $\mathrm{y}), \varphi$ is the angle between vector $\rho$ and the $\mathrm{x}$-axis in a counter clockwise direction and $R_{q, p}(\rho)$ is the Zernike radial polynomial.

The Zernike radial polynomials, $R_{q, p}(\rho)$, are defined as Eq. 2:

$$
\begin{aligned}
& \mathrm{R}_{\mathrm{q}}^{\mathrm{p}}(\rho)=\sum_{1=|\mathrm{p}| \mathrm{q}-\mathrm{l}=\mathrm{even}}^{\mathrm{q}} \frac{(-1)^{\frac{\mathrm{q}-1}{2}} \frac{\mathrm{q}+1}{2} !}{\frac{(\mathrm{q}-1)}{2} ! \frac{1+\mathrm{p}}{2} ! \frac{1-\mathrm{p}}{2} !} \rho^{1} \\
& \mathrm{R}_{\mathrm{q}}^{\mathrm{p}}(\rho)=\sum_{1=|\mathrm{p}| \mathrm{q}-\mathrm{l}=\mathrm{even}}^{\mathrm{q}} \beta_{\mathrm{q}, \mathrm{p}, \rho^{1}}
\end{aligned}
$$

Here:

$$
R_{q, p}(\rho)=R_{q-p}(\rho)
$$

From Eq. 1 the bases functions are orthogonal, thus satisfying Eq. 3 and 4: $\frac{\mathrm{a}+1}{\pi} \iint_{\mathrm{x}^{2}+\mathrm{y} 2 \leq 1} \mathrm{~V}_{\mathrm{p}, \mathrm{q}}(\mathrm{x}, \mathrm{y}) \mathrm{V}_{\mathrm{m}, \mathrm{n}}^{*}(\mathrm{x}, \mathrm{y})=\delta_{\mathrm{p}, \mathrm{m}} \delta_{\mathrm{q}, \mathrm{n}}$

Where:

$\delta_{\mathrm{a}, \mathrm{b}}=\left\{\begin{array}{cc}1 & \text { if } \mathrm{a}=\mathrm{b} \\ 0 & \text { otherwise }\end{array}\right.$

The Zernike moment of order $q$ with repetition $p$ for a digital image function $\mathrm{f}(\mathrm{x}, \mathrm{y})$ is given by Eq. 5:

$\mathrm{Zq}, \mathrm{p}=\frac{\mathrm{q}+1}{\pi} \Sigma \Sigma_{\mathrm{x}^{2}+\mathrm{y}^{2} \leq 1} \mathrm{f}(\mathrm{x}, \mathrm{y}) \mathrm{V}_{\mathrm{q}, \mathrm{p}}^{*}(\mathrm{x}, \mathrm{y})$

where, $\mathrm{V}_{\mathrm{q}, \mathrm{p}}^{*}(\mathrm{x}, \mathrm{y})$ is the complex conjugate of $\mathrm{V}_{\mathrm{q}, \mathrm{p}}(\mathrm{x}$, $\mathrm{y})$. In order to compute the Zernike moments of a given image, the origin is assumed to be the center of mass. The function $\tilde{\mathrm{f}}(\mathrm{x}, \mathrm{y})$ can be represented as Eq. 6 :

$\tilde{f}(x, y)=\sum_{q=0}^{N} \frac{C_{q, 0}}{2} R_{q, 0}(\rho)+\sum_{q=1}^{N} \sum_{p>0}$
$\left(C_{q, p} \cos p \varphi+S_{q, p} \sin p \varphi\right) R_{q, p}(\rho)$

where, $\mathrm{N}$ is the maximum order of Zernike moments, $\mathrm{C}_{\mathrm{q}, \mathrm{p}}$ is the real part of the $\mathrm{Z}_{\mathrm{q}, \mathrm{p}}$ and $\mathrm{S}_{\mathrm{q}, \mathrm{p}}$ denotes the imaginary part of $\mathrm{Z}_{\mathrm{q}, \mathrm{p}}$ :

To compute higher order Zernike moments, it is expensive and too difficult. In order to reduce the computational complexity common terms in Zernike moments are detected with different order and repetition. Specifically, by substituting Eq. 2 and 1 in 5 and re-organizing the terms the Zernike moments can be calculated as follows.

Moments can be calculated in the following form:

$$
\begin{aligned}
& \left.\mathrm{Z}_{\mathrm{q}, \mathrm{p}}=\frac{\mathrm{q}+1}{\pi} \sum \sum_{\mathrm{x} 2+\mathrm{y} 2 \leq 1} \sum_{\mathrm{l}=|\mathrm{p}|}^{\mathrm{q}} \beta \mathrm{q}, \mathrm{p}, 1 \rho^{1}\right) \mathrm{e}--^{\mathrm{jp} \varphi} \mathrm{f}(\mathrm{x}, \mathrm{y}) \\
& \mathrm{Z}_{\mathrm{q}, \mathrm{p}}=\frac{\mathrm{q}+1}{\pi} \sum_{\mathrm{l}=|\mathrm{p}|}^{\mathrm{q}} \beta_{\mathrm{q}, \mathrm{pl}}\left(\sum_{\mathrm{x} 2+\mathrm{y} 2 \leq 1} \mathrm{e}-{ }^{\mathrm{jp} \varphi \mathrm{pl}} \mathrm{f}(\mathrm{x}, \mathrm{y})\right) \\
& \mathrm{Z}_{\mathrm{q}, \mathrm{p}}=\frac{\mathrm{q}+1}{\pi} \sum_{1=|\mathrm{p}|}^{\mathrm{q}} \beta_{\mathrm{q}, \mathrm{p}, 1} \mathrm{x}_{\mathrm{p}, 1}
\end{aligned}
$$

The $\chi_{\mathrm{p}, 1}$ given in Eq. 7 is the common term in the computation of Zernike moments with the same repetition as shown in Fig. 1; for the case of repetition $\mathrm{m}=0$. Table 1 demonstrates the common terms to compute Zernike moments up to order N. Table 1 illustrates all the $\chi \mathrm{p}, 1$ to be computed for each repetition up to order 10 . 
Am. J. Applied Sci., 9 (5): 759-765, 2012

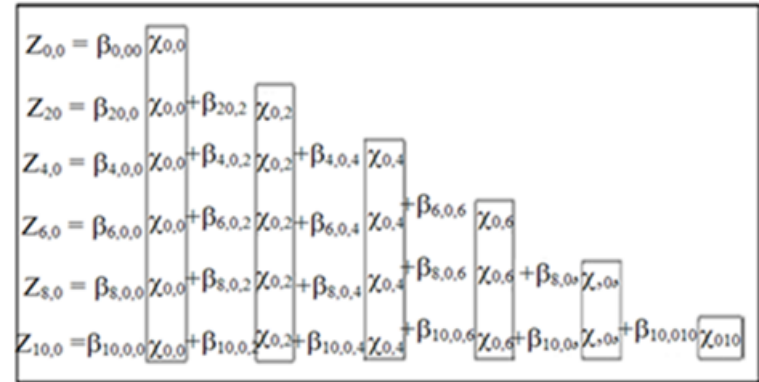

Fig.1: The common terms to compute Zernike moments up to 10 orders with zero repetition

Table1: $\chi_{\mathrm{p}, \mathrm{l}}$ Computation to compute zernike moments up to order 10 and repetition $\mathrm{p}$

\begin{tabular}{ll}
\hline Repetition $\mathrm{p}$ & $\chi_{\mathrm{p}, 1}$ \\
\hline 0 & $\chi_{0,0}, \chi_{0,2}, \chi_{0,4}, \chi_{0,6}, \chi_{0,8}, \chi_{0,10}$ \\
1 & $\chi_{1,1}, \chi_{1,3}, \chi_{1,5}, \chi_{1,7}, \chi_{1,9}$ \\
2 & $\chi_{2,2}, \chi_{2,4}, \chi_{2,6}, \chi_{2,9}, \chi_{2,10}$ \\
3 & $\chi_{3,3}, \chi_{3,5}, \chi_{3,7}, \chi_{3,9}$ \\
4 & $\chi_{4,4}, \chi_{4,6}, \chi_{4,8}, \chi_{4,10}$ \\
5 & $\chi_{5,5}, \chi_{5,7}, \chi_{5,9}$ \\
6 & $\chi_{6,6}, \chi_{6,8}, \chi_{6,10}$ \\
7 & $\chi_{7,7}, \chi_{7,9}$ \\
8 & $\chi_{8,8}, \chi_{8,10}$ \\
9 & $\chi_{9,9}$ \\
10 & $\chi_{10,10}$ \\
\hline
\end{tabular}

The second row of the table corresponds to the $\chi \mathrm{p}, 1$ shown in Fig. 1. Once all the entries in Table 1 are computed, Zernike moments with any order and repetition can be calculated as a linear combination of $\chi \mathrm{p},{ }_{1}$ as shown in Eq. 7. Also, note that the coefficients $\beta_{\mathrm{q}, \mathrm{p}, 1}$ are independent of the image or the coordinates; therefore, they can be stored on a small lookup table to save further computation.

The properties of Zernike moment are as follows:

There are even and odd values of Zernike polynomials Eq. 8 From Eq. 1 we can incur that even and odd Zernike polynomials can be written as follows:

$Z_{\mathrm{q}}^{\mathrm{p}}(\rho, \varphi)=\mathrm{R}_{\mathrm{q}}^{\mathrm{p}}(\rho) \cos (\mathrm{p}, \varphi)$

$Z_{q}^{-p}(\rho, \varphi)=R_{q}^{p}(\rho)$

Where:

$\mathrm{p}$ and $\mathrm{q}=$ Nonnegative integers with $\mathrm{q}>\mathrm{p}$

$\varphi \quad=$ The azimuthal angle

$\rho \quad=$ The radial distance $0 \leq \rho \leq 1$

The radial polynomials $\mathrm{R}_{\mathrm{q}}^{\mathrm{p}}$ are defined by Eq. 9:

$\mathrm{R}_{\mathrm{q}}^{\mathrm{p}}(\rho)=\sum_{1=0}^{\left(\frac{\mathrm{q}-\mathrm{p}}{2}\right)} \frac{(-1)^{1}(\mathrm{q}-1) !}{1 !\left(\frac{\mathrm{q}+\mathrm{p}}{2}\right) !\left(\frac{\mathrm{q}-\mathrm{p}}{2}-1\right) !} \rho^{\mathrm{q}-21}$
The orthogonality in the radial part is given by Eq. 10 as follows:

$$
\mathrm{f}_{0}^{1} \rho \sqrt{(2 \mathrm{q}+2)} \mathrm{R}_{\mathrm{q}}^{\mathrm{p}}(\rho) \sqrt{\left(2 \mathrm{q}^{\prime}+2\right)} \mathrm{R}_{\mathrm{q}^{\prime}}^{\mathrm{p}}(\rho) \mathrm{d} \rho=\delta_{\mathrm{qq}^{\prime}}
$$

Orthogonality in the angular part is represented by Eq. 11:

$$
\begin{aligned}
& \int_{0}^{2 \pi} \cos (\mathrm{p} \varphi) \cos \left(\mathrm{p}^{\prime} \varphi\right) \mathrm{d} \varphi=\epsilon_{\mathrm{p}} \pi \delta|\mathrm{p}|,\left|\mathrm{p}^{\prime}\right| \\
& \int_{0}^{2 \pi} \sin (\mathrm{p} \varphi) \sin \left(\mathrm{p}^{\prime} \varphi\right) \mathrm{d} \varphi=(-1)^{\mathrm{p}+\mathrm{q}^{\prime}} \pi \delta|\mathrm{p}|,\left|\mathrm{p}^{\prime}\right| \text { Where } \mathrm{p} \\
& \quad \neq 0
\end{aligned}
$$

$\int_{0}^{2 \pi} \cos (\mathrm{p} \varphi) \cos \left(\mathrm{p}^{\prime} \varphi\right) \mathrm{d} \varphi=0$

As orthogonal frequently appears in conjunction with Bessel functions it is also called the Neumann factor (Kintner, 1976). The product of the angular and radial parts establishes the orthogonality of the Zernike functions with respect to both indices if integrated over the unit disk Eq. 12:

$\int Z_{\mathrm{q}}^{\mathrm{p}}(\rho, \varphi) Z_{\mathrm{q}^{\prime}}^{\mathrm{p}^{\prime}}(\rho, \varphi) \mathrm{d}^{2} \mathrm{r}=\frac{\in \mathrm{p} \pi}{2 \mathrm{q}+2} \delta_{\mathrm{q}}, \delta_{\mathrm{p}, \mathrm{p}^{\prime}}$

where, $d^{2}=r d r d \varphi$ defined as Jacobian of the circular coordinate system and where q-p and q'-p' are both even. A special value is $\mathrm{R}_{\mathrm{q}}^{\mathrm{p}}(1)=1$ :

The parity with respect to reflection along the $\mathrm{x}$ axis is given by Eq. 13:

$Z_{\mathrm{q}}^{ \pm p}(\rho, \varphi)= \pm Z_{\mathrm{q}}^{ \pm \mathrm{p}}(\rho, \varphi)$

The parity with respect to point reflection at the center of coordinates is:

$$
Z_{\mathrm{q}}^{\mathrm{p}}(\rho, \varphi)=(-1)^{\mathrm{p}} \mathrm{Z}_{\mathrm{q}}^{\mathrm{p}}(\rho, \varphi+\pi)
$$

where, $(-1)^{\mathrm{p}}$ could as well be written $\left(-1^{) \mathrm{q}}\right.$ because $\mathrm{q}-\mathrm{p}$ is even for the relevant, non-vanishing values. The radial polynomials are also either even or odd is given by Eq. 14:

$$
\mathrm{R}_{\mathrm{q}}^{\mathrm{p}}(\rho)=(-1)^{\mathrm{p}} \mathrm{R}_{\mathrm{q}}^{\mathrm{p}}(-\rho)
$$

The periodicity of the trigonometric functions implies invariance if rotated by multiples of $2 \pi / \mathrm{m}$ radian around the center which is stated by Eq. 15: 
Am. J. Applied Sci., 9 (5): 759-765, 2012

$$
\mathrm{Z}_{\mathrm{q}}^{\mathrm{p}}\left(\rho, \varphi+\frac{2 \pi \mathrm{l}}{\mathrm{m}}\right)=\mathrm{Z}_{\mathrm{q}}^{\mathrm{p}}(\rho, \varphi), 1=0, \pm 1, \pm 2
$$

The features of the image are extracted using Zernike polynomial and the resultant is Zernike moment of the image.

$$
\mathrm{R}_{\mathrm{q}}^{\mathrm{p}}(\rho) \sum_{\mathrm{l}=0}^{\frac{\mathrm{q}-\mathrm{p}}{2}} \frac{(-1) 1(\mathrm{q}-1) !}{\left(\frac{\mathrm{q}+\mathrm{p}}{2}-1\right) !\left(\frac{\mathrm{q}-\mathrm{p}}{2}-1\right) !} \rho^{\mathrm{q}-21}
$$

where, $\mathrm{p}$ and $\mathrm{q}$ are the dimensions of the image considered for the operation. Here, 1 is the order of the moment. In our experiment we consider order $1=4$ The total computational cost for Zernike moments of order up to the Nth is:

$$
\frac{1}{960(\mathrm{~N} 2)(\mathrm{N}+1)(\mathrm{N} 3+132 \mathrm{~N} 2+1387 \mathrm{~N}+3240)} \text { for multiplication }
$$

$\frac{1}{960(\mathrm{~N}+2)(\mathrm{N}+1)(\mathrm{N} 3+92 \mathrm{~N} 2+947 \mathrm{~N}+2280)}$ for additions

The features are obtained by calculating the magnitude of the image by finding the moment. The values are normalized before classification. Due to its orthogonality we can use Zernike moment for image feature extraction and reconstruction of image.

Feature that can be extracted using Zernike moment (Chora's, 2007).

General features: Application independent features such as color, texture and shape. According to the abstraction level, they can be further divided into the following:

- Pixel-level features: Features calculated at each pixel, e.g., color, location

- Local features: Features calculated over the results of subdivision of the image band on image segmentation or edge detection

- Global features: Features calculated over the entire image or just regular sub-area of an image

Domain-specific features: Application dependent features such as human faces, fingerprints and conceptual features. These features are often a synthesis of low-level features for a specific domain. The fingerprints line and palm print can be observed at particular axis which can be used for matching the points with the test image.

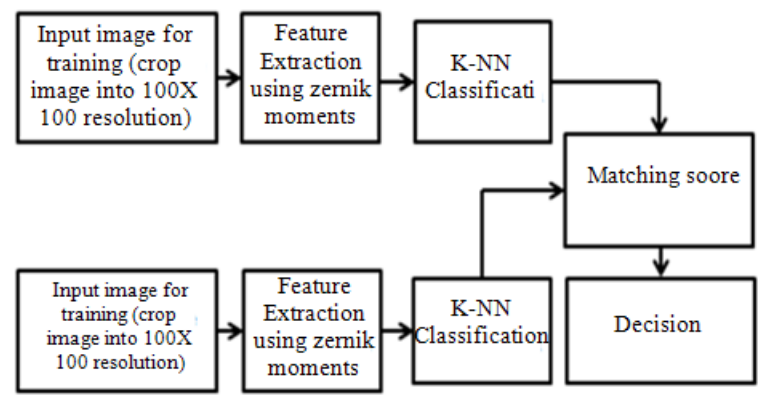

Fig. 2: Block diagram of the proposed design

Table 2: Comparision

\begin{tabular}{lll}
\hline $\begin{array}{l}\text { Cateogories } \\
\text { rate }\end{array}$ & Sample number & Recognition \\
\hline $\begin{array}{l}\text { Identification based } \\
\text { on gabor transform }\end{array}$ & 120 & $93.30 \%$ \\
$\begin{array}{l}\text { Wavalet transform } \\
\text { Lu } \text { et al. } \text { (2006) }\end{array}$ & 50 & $96.30 \%$ \\
$\begin{array}{l}\text { Hierarchical method } \\
\text { Boiman } \text { et al. }(2008)\end{array}$ & 386 & $97.80 \%$ \\
This method & 125 & $98 \%$ \\
\hline
\end{tabular}

The moment function of the 2-D image is used in object recognition and identification of images. Zernike moment function is effectively used in the pattern recognition of palm print images as their rotational invariants can be easily constructed (Table 2).

Proposed design description: Figure 2 shows overall framework of the proposed palmprint biometric authentication. From Fig. 2, it can be seen that the moment features are extracted and classified using $\mathrm{K}$ Nearest Neighbourhood algorithm.We use K-NN algorithm to find the matching score between the test image and the original image stored in the database.

- The images are stored in a database folder

- Images are read one by one and features are extracted using Zernike moment.

- The features are normalized and stored in a variable for training and testing.

- The classification algorithm is called to plot the cluster graph of test and train image

- We can find the matching score by subtracting the value of the reconstructed images between the train and test image.

- The clusters also show the particular cluster of train images where the test image belongs.

- The scoring value is either zero or very close to zero.

- We chose the score which is exactly equal to zero or the value which is nearest to zero.

- The matched image output is obtained from the group of train images by determining the scoring value. 


\section{Algorithm:}

1) Take an image window and place it in a squared window such that its size is maximized without distortion.

2) First create a square of size max (width, height) and put the original image into the center.

3) Resize the square image to a size smaller than the desired size in order to take into account the spherical nature (disk!) of the Zernike moments.

4) Now create a black square of the desired size and put the created image into its center

5) Converts the elements of an array into unsigned 8bit (1-byte) integers

6) Calculate the Zernike basis functions up to an order for a square image of size size (rows=cols!)

7) All pixels out of the disk inscribed into the image square are ignored.

8) Create a structure consisting of

a. The Zernike basis functions (image Size, order of moment, with negative or not).

9) Calculate the Zernike moments from the palmprint image. The COMPLEX Zernike moments of the palmprint image is computed using the Zernike basis functions. Iterate through the moments, beginning from the lowest to the highest

10) Reconstruct an palmprint image from some of its Zernike moments. We suppose that some amount of first (lower) order moments, not necessarily all moments, are available

11) The required data to reconstruct image are as follows:-

a. The complex zernike moments

12) The size of the square image

13) The Zernike basis functions must be recomputed to derive lower order polynomials

The image can be classified using reconstructed image value. The difference between the moment values give the similarity count which can be used in the classification.

The classification is the grouping of the cluster of images between the test image and train image. The mean distance between the centroid of the train image and the test image is calculated. The nearest point is chosen and plots the value which forms a cluster. The distance calculation is based on Euclidean distance weight function. If the value is too far it is not considered.

In 2-D, the Euclidean distance (Hu et al., 1962) between $(\mathrm{x} 1, \mathrm{y} 1)$ and $(\mathrm{x} 2, \mathrm{y} 2)$ is given by Eq. 16:

$$
\sqrt{(x 1-x 2)^{2}}+(y 1-y 2)^{2}=c
$$

Euclidean distance algorithm of classification is non-parametric as their classification is directly dependent on the data Boiman et al. (2008). The objects are trained according to the data and the test image can be classified using the same process as the object or image was trained.

Non-parametric classifiers have several very important advantages that are not shared by most learning-based approaches:

- Can naturally handle a huge number of classes

- Avoid over fitting of parameters, which is a central issue in learning based approaches

- Require no learning/ training phase. Although training is often viewed as a one-time preprocessing step, retraining of parameters in large dynamic databases may take days, whereas changing classes/training-sets is instantaneous in nonparametric classifiers

The nearest neighbor classifier Boiman et al. (2008) relies on a metric or a distance function between points. For all points $\mathrm{x}, \mathrm{y}$ and $\mathrm{z}$, a metric $\mathrm{T}(\mathrm{x}, \mathrm{y}, \mathrm{z})$ must satisfy the following properties:

- No negativity : $\mathrm{T}(\mathrm{x}, \mathrm{y}) \geq 0$

- Reflexivity : $\mathrm{T}(\mathrm{x}, \mathrm{y})=0$ if and only if $\mathrm{x}=\mathrm{y}$

- $\quad$ Symmetry : $\mathrm{T}(\mathrm{x}, \mathrm{y})=\mathrm{T}(\mathrm{y}, \mathrm{x})$

- $\quad$ Triangle inequality : $\mathrm{T}(\mathrm{x}, \mathrm{y})+\mathrm{T}(\mathrm{y}, \mathrm{z}) \geq \mathrm{T}(\mathrm{x}, \mathrm{z})$

The nearest neighbor classifier is used to compare the feature vector of the prototype image and feature vectors stored in the database. It is obtained by finding the distance between the prototype image and the database. Let $\mathrm{C}_{11}, \mathrm{C}_{21}, \mathrm{C}_{31} \ldots \mathrm{C}_{\mathrm{k} 1}$ be the $\mathrm{k}$ clusters in the database. The class is found by measuring the distance $\mathrm{T}\left(\mathrm{x}(\mathrm{q}), \mathrm{C}_{\mathrm{k}}\right)$ between $\mathrm{x}(\mathrm{q})$ and the $\mathrm{k}^{\text {th }}$ cluster $\mathrm{C}^{\mathrm{k} 1}$. The feature vector with minimum difference is found to be the closest matching vector. It is given by Saradha and Annadurai (2005):

$$
\mathrm{T}\left(\mathrm{x}(\mathrm{q}), \mathrm{C}_{\mathrm{K}}\right)=\min \left\{\|\mathrm{x}(\mathrm{q})-\mathrm{x}\|: \mathrm{x} \in \mathrm{C}_{\mathrm{k}}\right.
$$

Nearest-neighbor classifiers provide good image classification when the query image is similar to one of the labeled images in its class.

\section{RESULTS AND DISCUSSION}

To evaluate the effectiveness of our proposed palm print Zernike moment based authentication scheme, a database containing palmprint samples is required. For our research, we use PolyU Palmprint Database, collected by the Biometric Research Center at The Hong Kong Polytechnic University, which is a widely used database in palmprint research. 

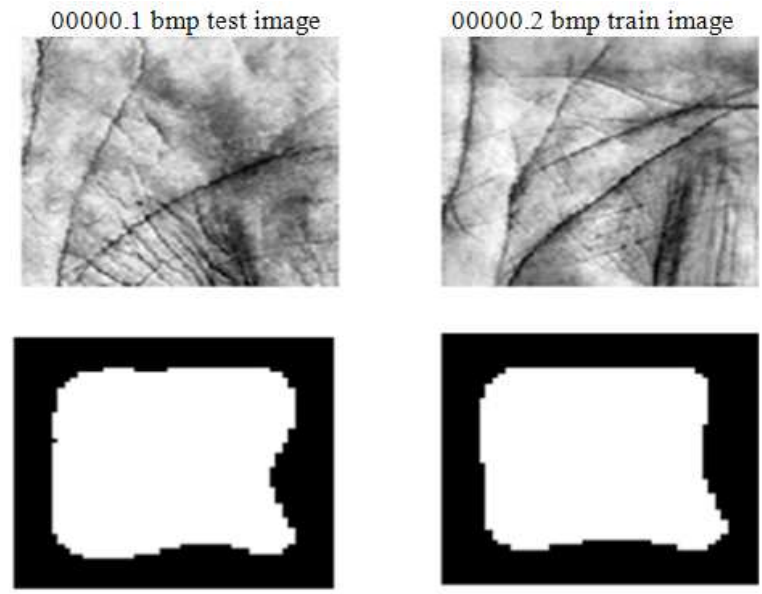

Difference: 64.000000

not matched

Fig. 3: Zernike Moments calculation and matching result

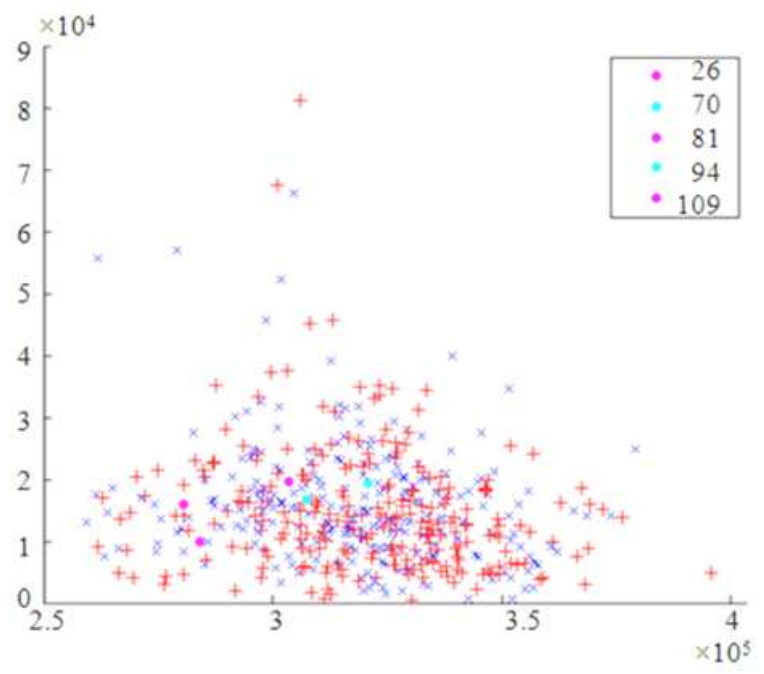

Fig. 4: Result of KNN classification for the Palmprint images

The database contains 7752 greyscale images, corresponding to 386 different palms with 20-21 samples for each in bit- map image format. We selected 125 individuals' left- hand palm images; every person has 5 and totaling up to 625 . We then get every person's palm images as a template (totaling 125). The remaining 500 are used as training samples. The experiments are conducted in MATLAB with image processing Toolbox and on a machine with an Intel core 2 Duo CPU processor. The test database has 125 different untrained images that undergo the same algorithm as trained image and we compare this to the original trained image.

Zernike moments at high orders are calculated from the images and the image is classified using $\mathrm{KNN}$. This algorithm is more effective as it has some advantages: orthogonallity and rotation invariance property.

Here the computational cost is reduced by detecting the common terms of Zernike moments. These characteristics are able to minimize information redundancy and provide geometrical invariance.

Figure 3 represents the calculation of Zernike moments and shows the difference between two images. If the difference between two images is zero, it means there is a perfect match. If the difference between two images is high, it indicates that the images do not match. Figure 4 shows the KNN classification of palmprint image. Out of 125 images, 5 images of different persons are taken for testing.

The colored circles are the test image representations. We designed and implemented an automated palmprint authentication system by applying a novel feature extraction and by matching images using high order Zernike moment invariants.

We designed and implemented an automated palmprint authentication system by applying a novel feature extraction and by matching images using high order Zernike moment invariants. Zernike moments at high orders are calculated from the images and the image is classified using KNN. This algorithm is more effective as it has some advantages: orthogonallity and rotation invariance property. Here the computational cost is reduced by detecting the common terms of Zernike moments. These characteristics are able to minimize information redundancy and provide geometrical invariance. The proposed palmprint authentication system achieves a recognition accuracy of $98 \%$ and interesting working point with False Acceptance Rate (FAR) of $=1.062 \%$ and False Rejection Rate (FRR) of $=0 \%$. The experimental results demonstrate the effectiveness of the proposed approach for palmprint authentication using Zernike moments compared with conventional palmprint recognition algorithms.

\section{CONCLUSION}

Computational cost can be reduced by detecting the common term of Zernike moment. Experiments and classifications have been performed using Hong Kong PolyU palm print database with 125 individuals' left hand palm images; every person has 5 samples, totaling up to 625 . We then get every person's palm images as a template (totaling 125). The remaining 500 are used as the training samples. The proposed palmprint authentication system achieves a recognition accuracy of $98 \%$ and interesting working point with False Acceptance Rate (FAR) of $=1.062 \%$ and False Rejection Rate (FRR) of $=0 \%$. Experimental 
evaluation demonstrates the efficient recognition performance of the proposed algorithm compared with conventional palmprint recognition algorithms.

\section{REFERENCES}

Al-Hamami, A.H. and S.A.A. Ani, 2005. A new approach for authentication technique. J. Comput. Sci., 1: 103-106. DOI: 0.3844/jcssp.2005.103.106

Basha, A.J., V. Palanisamy and T. Purusothaman, 2011. Efficient multimodal biometric authentication using fast fingerprint verification and enhanced iris features. J. Comput. Sci., 7: 698-706.DOI: 10.3844/jcssp.2011.698.706

Boiman, O., E. Shechtman and M. Irani, 2008. In Defense of Nearest-Neighbor Based Image Classification. Porceedings of the IEEE Conference on Computer Vision and Pattern Recognition (CVPR), June 23-28, IEEE Xplore Press, Anchorage, pp: 1-8. DOI: 10.1109/CVPR.2008.4587598

Breu, H., J. Gil, D. Kirkpatrick and M. Werman, 1995. Linear time euclidean distance transform algorithms. IEEE Trans. Patt. Anal. Mach. Intel., 17: 529-533. DOI: 10.1109/34.391389

Chora's, R.S. 2007. Image feature extraction techniques and their applications for cbir and biometrics systems. Int. J. Biol. Bio. Eng., 1: 6-16.

Gu, J., H.Z. Shu, C. Toumoulin and L.M. Luo, 2002. A novel algorithm for fast computation of Zernike moments. Patt. Recog., 35: 2905 2911. DOI: 10.1016/S0031-3203(01)00194-7

Hu , M.K., 1962. Visual pattern recognition by moment invariants. IRE Trans. Inform. Theory, 8: 179-187. DOI: 10.1109/TIT.1962.1057692

Khotanzad, A.Y.H., 1990. Invariant image recognition by Zernike moments. IEEE Trans. Patt. Anal. Mach. Intell., 12: 489-497. DOI: 10.1109/34.55109

Kintner, E.C., 1976. On the mathematical properties of the Zernike Polynomials. Opt. Acta 23: 679-680. DOI: $10.1080 / 713819334$
Krishneswari, K. and S. Arumugam, 2012. Multimodal biometrics using feature fusion. J. Comput. Sci., 8: 431-435. DOI: 10.3844/jcssp.2012.431.435

Lu,Y., L. Jiang, J. Kong, Y. Wang and Y. Li, 2006. An Adaptive and robust hand-based personal identification. Proceedings of the IEEE Mediterranean Electrotechnical Conference, May 16-19, IEEE Xplore Press, Malaga, pp: 494-497. DOI: 10.1109/MELCON.2006.1653146

MeenakshiDevi, P., M. Venkatesan and K. Duraiswamy, 2009. A fragile watermarking scheme for image authentication with tamper localization using integer wavelet transform. J. Comput. Sci., 5: 831-837. DOI: 10.3844/jcssp.2009.831.837

Mukundan, R. and K.R. Ramakrishnan, 1995. Fast computation of Legendre and Zernike moments. Patt. Recog., 28: 1433 1442. DOI: 10.1016/00313203(95)00011-N

Prokop, R.J. and A.P. Reeves, 1992. A survey of moment-based techniques for unoccluded object representation and recognition. Graph. Models Image Process CVGIP, 54: 438 460. DOI: 10.1016/1049-9652(92)90027-U

Saradha, A. and S. Annadurai, 2005. A Hybrid feature extraction approach for face recognition systems. ICGST-GVIP J., 5: 23-30.

Teague, M.R., 1980. Image analysis via the general theory of moments. J. Opt. Soc. Am, 70: 920930. DOI: $10.1364 / \mathrm{JOSA} .70 .000920$

Teh, C.H. and R.T. Chin, 1988. On image analysis by the methods of moments. IEEE Transactions on Pattern Analysis and Machine Intell. 10: 496-513. DOI: $10.1109 / 34.3913$ 